\section{Heterochromatin establishment at pericentromeres depends on nuclear position}

\author{
Joanna W. Jachowicz, ${ }^{1,3}$ Angèle Santenard,, ${ }^{1,3}$ \\ Ambre Bender, ${ }^{1}$ Julius Muller, ${ }^{2}$ \\ and Maria-Elena Torres-Padilla ${ }^{1,4}$ \\ ${ }^{1}$ Institut de Génétique et de Biologie Moléculaire et Cellulaire, \\ CNRS/INSERM U964, Université de Strasbourg, F-67404 \\ Illkirch, France; ${ }^{2}$ Laboratory of Chromatin, Epigenetics, and \\ Differentiation, Institute of Molecular and Cell Biology, Agency \\ for Science, Technology, and Research, Singapore 138673, \\ Singapore
}

Mammalian development begins with fertilization of an oocyte by the sperm followed by genome-wide epigenetic reprogramming. This involves de novo establishment of chromatin domains, including the formation of pericentric heterochromatin. We dissected the spatiotemporal kinetics of the first acquisition of heterochromatic signatures of pericentromeric chromatin and found that the heterochromatic marks follow a temporal order that depends on a specific nuclear localization. We addressed whether nuclear localization of pericentric chromatin is required for silencing by tethering it to the nuclear periphery and show that this results in defective silencing and impaired development. Our results indicate that reprogramming of pericentromeric heterochromatin is functionally linked to its nuclear localization.

Supplemental material is available for this article.

Received June 16, 2013; revised version accepted October 16, 2013.

In mammals, fertilization of an oocyte by the sperm is followed by epigenetic reprogramming, which involves de novo acquisition of chromatin signatures in the two parental genomes, but the molecular determinants underlying such reprogramming are not fully understood. In particular, the formation of heterochromatin de novo is thought to be essential to ensure the subsequent organization of the embryonic epigenome and embryonic development (Probst and Almouzni 2011; Fadloun et al. 2013; Nestorov et al. 2013).

In the zygote, remodeling of the paternal chromatin is particularly extensive, since it is subject to a nearly genome-wide replacement of protamines by maternally supplied histones. A few hours after fertilization, the pericentromeric chromatin must acquire a highly compact

[Keywords: pericentromeric chromatin; nuclear organization; epigenetic reprogramming; heterochromatin]

${ }^{3}$ These authors contributed equally to this work.

${ }^{4}$ Corresponding author

E-mail metp@igbmc.fr

Article is online at http://www.genesdev.org/cgi/doi/10.1101/gad.224550.113. chromatin organization for the first time to allow subsequent kinetochore loading and progression through the first mitosis. The pericentromere domain is formed by tandem repeats of major and minor satellite repeats, which constitute the pericentric and centric chromatin, respectively. In the embryo, the initial silencing of pericentromeric chromatin requires the Lys27 of the histone variant $\mathrm{H} 3.3$ and is accompanied by progressive acquisition of $\mathrm{H} 3 \mathrm{~K} 27$ methylation and tethering of $\mathrm{HP} 1 \beta$ in a mechanism that involves a burst of transcription from the major satellite (MajSat) repeats in the zygote, in analogy to heterochromatin formation in Schizosaccharomyces pombe (Grewal and Elgin 2007; Puschendorf et al. 2008; Probst et al. 2010; Santenard et al. 2010). This heterochromatinization in mammals, however, seems to be "atypical," since it is independent of H3K9me3 and H4K20me3, which are absent from the paternal chromatin (Arney et al. 2002; Kourmouli et al. 2004; Santos et al. 2005). In agreement, the expression of an H3.3K27R mutant disturbs heterochromatin silencing at the pericentromeres, while expression of an H3.3K9R mutant has no discernible effect in development (Santenard et al. 2010).

Pericentromeric heterochromatin is typically visualized as DAPI-rich regions in mouse somatic cells in interphase, which are referred to as chromocenters. These form upon clustering of pericentromeric domains from several chromosomes. In the embryo, however, the pericentromeric chromatin does not form chromocenters until the late two-cell/early four-cell stage transition (Martin et al. 2006a; Probst et al. 2007; Aguirre-Lavin et al. 2012). Instead, the mammalian embryo displays a distinctive nuclear organization with a radial arrangement of the chromosome territories with centromeres attached to the nucleoli precursors. The pericentromeric chromatin envelops these precursors, which are referred to as nucleolar-like bodies (NLBs), forming a characteristic ring-like structure.

It is increasingly clear that nuclear organization provides a landmark for gene regulation (Akhtar and Gasser 2007; Kumaran et al. 2008). Although a number of studies have documented changes in the nuclear localization of pericentromeric chromatin after fertilization that correlate with reprogramming efficiency upon somatic cell nuclear transfer (Martin et al. 2006b), a functional role for this process in heterochromatin establishment and/or maintenance has not been addressed, and it is not known whether the spatial organization of the genome can regulate reprogramming. Here, we addressed the temporal dynamics of the acquisition of heterochromatic signatures of the pericentric chromatin in relation to their position in the nuclear three-dimensional (3D) space. We found that the pericentric repeats reach their localization around the nucleoli precursors in the zygote prior to the acquisition of their embryonic heterochromatic signatures (H3K27me1, H3K27me3, and HP1 $\beta)$, suggesting that their spatial configuration is essential for heterochromatic silencing. We tested this hypothesis by artificially

(C) 2013 Jachowicz et al. This article is distributed exclusively by Cold Spring Harbor Laboratory Press for the first six months after the full-issue publication date (see http://genesdev.cshlp.org/site/misc/terms.xhtml). After six months, it is available under a Creative Commons License (Attribution-NonCommercial 3.0 Unported), as described at http:// creativecommons.org/licenses/by-nc/3.0/. 
tethering pericentromeric chromatin to the nuclear periphery using a designed zinc finger $(\mathrm{ZF})$ fused to emerin, an integral component of the nuclear envelope. Our results show that the spatial localization of the pericentromeric heterochromatin is essential for its silencing and the reprogramming of embryonic chromatin after fertilization.

\section{Results and Discussion}

We first addressed the temporal dynamics of the nuclear positioning of pericentromeric chromatin in relation to the acquisition of heterochromatic signatures on the MajSats after fertilization. DNA-FISH was incompatible with the analysis of histone modifications and HP1 $\beta$, since we noted that the immunostaining profile of several histone modifications and HP1 $\beta$ was perturbed after DNA-FISH as compared with immunostaining alone, presumably due to the denaturing conditions required for FISH with probes other than oligonucleotides (data not shown). Thus, to locate the pericentromeric chromatin in the embryo, we employed a GFP-tagged polydactyl ZF that recognizes specifically the MajSat sequences (see also below) (Lindhout et al. 2007). Importantly, the ZF-GFP showed the same pattern of localization of pericentromeric repeats as that obtained upon DNA-FISH with a MajSat probe, with a progressive decondensation from the center of the male pronucleus toward an organization into rings surrounding the NLBs, in agreement with previous reports (Fig. 1A,B; Martin et al. 2006a; Probst et al. 2007). The female pronucleus also showed the expected pattern of reorganization around the NLBs (Fig. 1A,B). A complete ring-like organization was clearly visible in early two-cell stage embryos (Fig. 1A,B).

While the female chromatin inherits H3K27 methylation from oogenesis, H3K27me3 is undetectable on the paternal pronucleus immediately after fertilization (Santos et al. 2005; Puschendorf et al. 2008; Santenard et al. 2010). Pericentromeric regions become localized in rings around the NLBs by the mid-zygotic stage, as judged from the ZF-GFP signal in pronuclear stage 2-3 (PN2-3) zygotes, without detectable levels of H3K27me3 (Fig. 1C). Remarkably, pericentromeric chromatin acquires $\mathrm{H} 3 \mathrm{~K} 27 \mathrm{me} 3$ after this stage, and H3K27me3 is clearly and sharply detected around the NLBs at late pronuclear stages, colocalizing with the ZFGFP signal (Fig. 1C). H3K27me1 and HP1 $\beta$ showed a more dispersed localization pattern in zygotes, but the enrichment of both of these heterochromatic marks with pericentromeric chromatin was only detected at the latest pronuclear stages, after they reach their ring-like destination around the NLBs (Fig. 1D,E). Thus, pericentromeric chromatin becomes localized in sharp rings around NLBs prior to the acquisition of a detectable heterochromatic signature, suggesting that this specific nuclear localization might be a prerequisite for silencing.
To address whether nuclear positioning of pericentromeric chromatin is required for heterochromatin establishment and subsequent silencing, we aimed to manipulate the localization of the MajSat in the nucleus. For this, we designed an approach that would lead to tethering of the pericentromeric chromatin to the nuclear periphery by fusing the above-described ZF to emerin, a constituent of the nuclear membrane (for review, see Bengtsson and Wilson 2004). The targeting fusion protein ZF-Eme is expected to associate with the MajSats via the $\mathrm{ZF}$ interaction and with the nuclear lamina via the emerin. We chose the nuclear periphery owing to its known
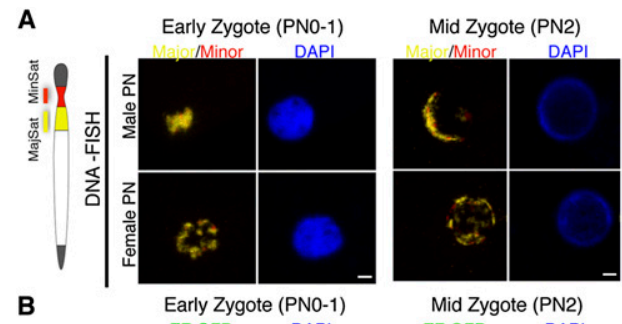

Mid Zygote (PN2)
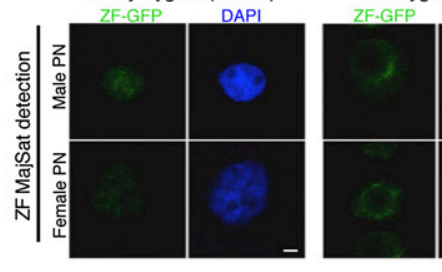

C

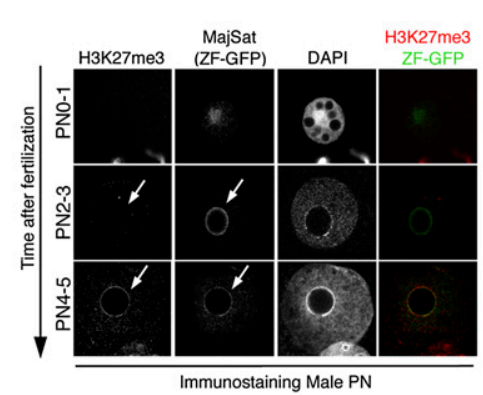

D

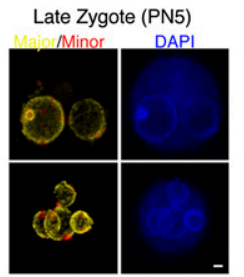

Late Zygote (PN5)
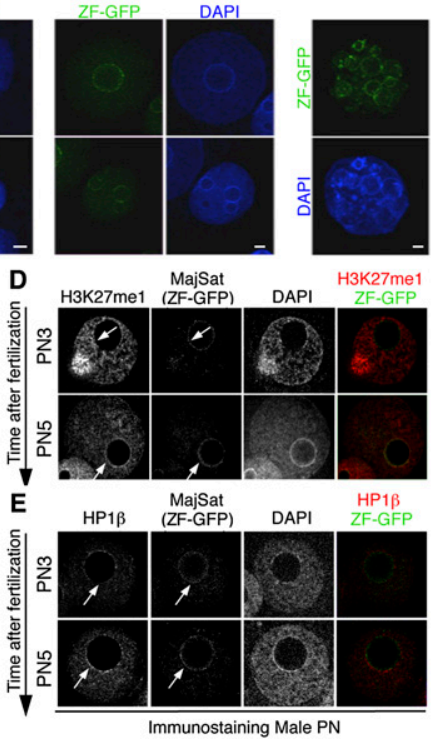

Figure 1. Pericentromeric chromatin localizes around the nucleoli prior to the acquisition of heterochromatic signatures. (A) DNA-FISH for major and minor satellite repeats. Representative zygotes immediately after fertilization (early, PN0-1) and at subsequent stages (mid and late) and early two-cell stage embryos are shown. Full projections of confocal sections of the male and female pronuclei are shown. (Left) A schematic representation of a mouse chromosome with centromeric regions and the probes color code. (PNO) pronuclear stage $0 ;(\mathrm{PN} 1)$ pronuclear stage 1 , etc. Bar, $2 \mu \mathrm{m}$. $(B)$ Temporal dynamics of the localization of pericentromeric chromatin using ZF design. mRNA coding a ZF targeting MajSats fused to GFP (ZFGFP) was microinjected at fertilization, and embryos were analyzed at the indicated times. Shown are maximal projections of confocal Z-series as in $A$. Note that the ZFGFP pattern recapitulates faithfully the pattern obtained by DNA-FISH (yellow label in A). Bar, $2 \mu \mathrm{m}$. (C) Localization of MajSats around the NLBs precedes acquisition of heterochromatic H3K27 methylation signatures on the paternal chromatin. Immunostaining of ZF-GFP-expressing embryos with an H3K27me3 antibody in early (PNO1), mid (PN2-3), and late (PN4-5) zygotes. Shown are representative single confocal sections of the male pronucleus with the H3K27me3, the GFP, and the DAPI channel in grayscale and the corresponding merge images. Arrows point to places of accumulation of MajSat repeats at the NLBs and the initial absence of H3K27me3 followed by strong accumulation of $\mathrm{H} 3 \mathrm{~K} 27 \mathrm{me} 3$ in late zygotes. (D) Immunostaining analysis as in $C$ but with an H3K27mel antibody. Note that the sharp signal of H3K27mel colocalizing with the ZF-GFP occurs only after ZF-GFP signal concentrates around the NLBs (arrows) in late (PN5) zygotes. (E) Accumulation of HP1 $\beta$ on the pericentromeric chromatin occurs after their relocation around the NLBs at later zygotic stages. Analysis as in $C$ and $D$ with an HP1 $\beta$ antibody. 
potential role in gene regulation and because it is the only other nuclear space that has been clearly identified in the embryo. Because chromatin immunoprecipitation (ChIP) analysis cannot be performed in the embryo, we used transiently transfected NIH3T3 as an additional control to verify binding of the ZF to the MajSats. The ZF-GFP fusion bound specifically to MajSat repetitive sequences-but not to SINE B1 or IAP elements-as demonstrated by ChIP analysis (Supplemental Fig. 1A). We then validated the ZF-Eme construct along with two negative controls-the ZF-GFP construct and a construct expressing emerin alone (HA-Eme)-in NIH3T3 cells. We confirmed by immunostaining that all three constructs were expressed in NIH3T3 cells. The ZF-GFP and HA-Eme controls localized to DAPI-rich regions and the nuclear periphery, respectively, as expected (Supplemental Fig. 1B). In contrast to the ZF-GFP construct, the ZF-Eme construct localized to the nuclear periphery (Supplemental Fig. 1B). Importantly, the ZF-Eme retained the specific ability to bind to MajSat sequences, in contrast to the emerin alone, which did not bind to the MajSat or any of the other repetitive sequences analyzed by ChIP (Supplemental Fig. 1C).

To address whether fusing the ZF to emerin results in efficient tethering of pericentromeric chromatin to the nuclear periphery, we performed 3D DNA-FISH with a MajSat probe in embryos expressing ZF-Eme. We microinjected mRNA for ZF-GFP or ZF-Eme immediately after fertilization and analyzed embryos at the twocell stage. While the ZF-GFP protein localized as expected around the NLBs and in the one to two chromocenters that are not associated to the NLBs (Probst et al. 2007), the ZF-Eme construct localized to the nuclear periphery in both nuclei of two-cell stage embryos (Fig. 2A). The localization pattern of the pericentromeric chromatin was severely affected in most ZF-Eme embryos and showed a spotty pattern with patches throughout the nuclear membrane rather than the ring and chromocenter configuration typically observed in two-cell stage
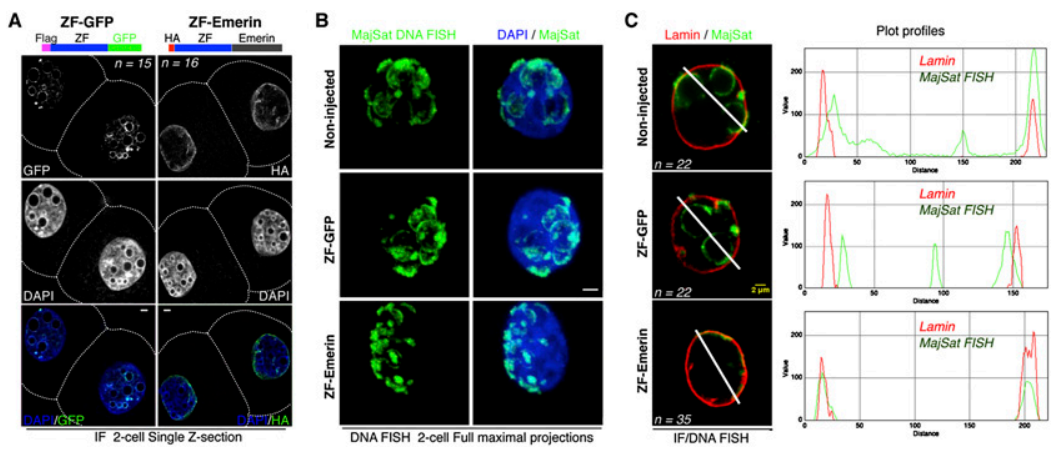

Figure 2. Expression of ZF-Eme results in efficient tethering of pericentromeric chromatin to the nuclear periphery in the early embryo. (A) Immunostaining of twocell stage embryos microinjected with mRNA for ZF-GFP and ZF-Eme using GFP acquisition or an HA antibody as indicated. Dotted lines delineate the cell membrane. Representative single confocal sections are shown. Bar, $2 \mu \mathrm{m}$. (B) Expression of ZF-Eme in embryos results in displacement of pericentromeric chromatin at the nuclear periphery. Embryos microinjected with the indicated mRNA were processed for 3D DNA-FISH with a MajSat probe (green); DNA was stained with DAPI (blue). Full Z-series projections are shown; serial confocal sections are shown in Supplemental Movies 1-3. Bar, $2 \mu \mathrm{m} .(C)$ Representative single confocal sections of immuno-DNA-FISH with a Lamin B antibody and a MajSat probe of two-cell stage embryos expressing the indicated proteins. The RGB profiles of the corresponding single confocal sections are shown at the right. Bar, $2 \mu \mathrm{m}$. control embryos (Fig. 2B; Supplemental Movies 1-3). Analysis of combined DNA-FISH for MajSat and immunostaining for endogenous Lamin B through single $\mathrm{Z}$ confocal sections revealed that pericentromeric chromaembryos (Fig. 2C), consistent with the localization of time point (Fig. 2A). While in control embryos, MajSats can be found both around the NLBs and close to the Lamin B domain (Fig. 2C), in ZF-Eme embryos, the proportion of MajSat signal not associated with the nuclear of MajSats to the nuclear periphery was time-sensitive, since expression of the same ZF-Eme construct at the late the MajSats toward the nuclear periphery at the four-cell controls (data not shown). Thus, our ing of the pericentromeric chromatin to the nuclear periphery.

We next asked whether altering the localization of ericentromeric chromatin results in defective heteroMajSats occurs primarily from the male pronucleus, and these transcripts are believed to be necessary for the subsequent silencing and organization into a somatic-like chromocenter configuration, which occurs progressively Probst et al. 2010; Santenard et al. 2010). We therefore (a) tin in two-cell stage embryos. Noninjected and ZF-GFP control embryos display a few transcription foci, as expected (Fig. 3A). In contrast, the ZF-Eme embryos displayed a significantly increased RNA-FISH signal, suggesting actively in ZF-Eme embryos as compared with controls (Fig. 3A). Quantification of both the number of MajSat transcription sites (Fig. 3B) and the volume of their transcripts (Fig. 3C) revealed that ZF-Eme embryos have a significantly higher transcriptional output of pericentromeric repeats, suggesting that repositioning of pericentromeric chromatin to the nuclear periphery leads to defective heterochromatin silencing. Moreover, displacing pericentromeric chromatin to the nuclear periphery also resulted in impaired chromocenter formation (Supplemental Fig. 2).

Next, we asked whether recruitment of pericentromeric chromatin to the nuclear periphery affects developmental progression. For this, we injected zygotes as before with ZF-GFP or HA-Eme mRNA as controls and ZF-Eme mRNA. For the two latter constructs, mRNA for GFP was coinjected as a positive control for microinjection. Embryos expressing HA-Eme and ZF-GFP developed at a similar rate and ratios compared with the noninjected control embryos, with between $83 \%$ and $88 \%$ embryos forming blastocysts, which are the routine values obtained in this type of manipulation (Fig. 3D; Supplemental Fig. 3A). In contrast, expression of ZF-Eme resulted in a significantly 
A

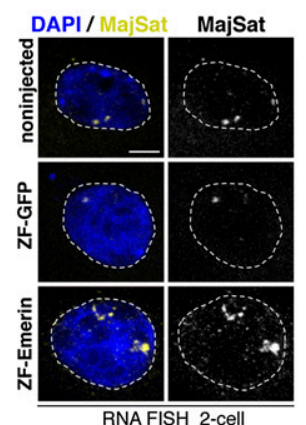

B

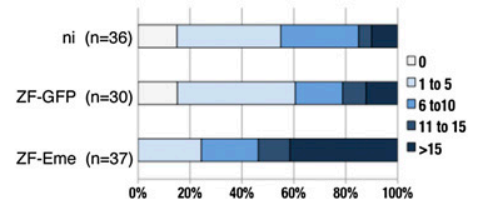

C

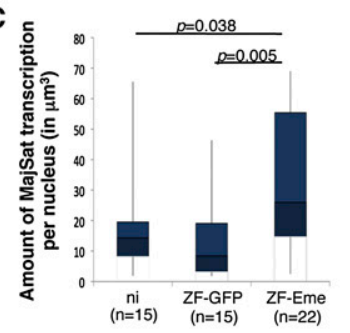

D

\begin{tabular}{r|c}
\multicolumn{1}{c|}{ Group } & $\begin{array}{c}\% \text { of embryos in } \\
\text { blastocyst stage }\end{array}$ \\
\hline ni (control) & $94 \%(n=59)$ \\
\hline HA-Emerin & $88 \%(n=40)$ \\
\hline ZF-GFP & $83 \%(n=29)$ \\
\hline ZF-Emerin & $51 \%(n=45)$ \\
& ${ }^{*} p=0.000001$
\end{tabular}

Figure 3. Tethering pericentromeric chromatin to the nuclear periphery impairs silencing and developmental progression. $(A)$ RNA-FISH to reveal nascent MajSat transcripts (yellow) in embryos expressing the indicated mRNAs. Representative nuclei at the twocell stage are shown. The dotted line demarcates the nuclear membrane. Bar, $5 \mu \mathrm{m}$. $(B)$ Quantification of the number of MajSat transcription sites as determined by RNA-FISH. Noninjected (ni) embryos or embryos expressing ZF-GFP or ZF-Eme were analyzed at the two-cell stage. Embryos were grouped according to the number of transcription sites per nucleus as indicated at the right (zero sites, between one and five sites, between six and 10 sites, etc.). The percentage of embryos belonging to each category was plotted for each expression construct. (C) Box plot of the quantification of the total volume of MajSats transcribed in noninjected (ni) embryos or embryos expressing ZF-GFP or ZF-Eme. (D) Analysis of developmental progression of noninjected embryos or embryos expressing HA-Eme, ZF-GFP, or ZF-Eme. Zygotes were microinjected with the indicated mRNAs. The number of embryos reaching the blastocyst stage for each group was scored after $3 \mathrm{~d}$ of development. $n$ indicates the total number of embryos analyzed per group.

reduced rate of developmental progression, with only $51 \%$ of the embryos reaching the blastocyst stage $(P=$ 0.000001 , Kruskal-Wallis test) (Fig. 3D). The remaining $49 \%$ stopped development between the two- and eightcell stages, similarly to embryos with defective heterochromatin silencing (Probst et al. 2010; Santenard et al. 2010). Importantly, the $51 \%$ of the embryos that did reach the blastocyst stage displayed delayed development. We also addressed whether embryos expressing ZF-Eme display division errors such as lagging chromatin. In line with our earlier findings of defective heterochromatin formation (Santenard et al. 2010), we observed a high incidence of lagging chromatin in ZF-Eme-expressing embryos $(21 \%$ compared with $6 \%, 3 \%$, and $7 \%$ for noninjected, ZF-GFP, and HA-Eme, respectively) (Supplemental Fig. 3B). Thus, targeting of endogenous pericentromeric chromatin to the nuclear periphery after fertilization impairs developmental progression. Whether this developmental phenotype is solely due to a silencing defect of pericentromeric chromatin and/or subsequent defects on kinetochore loading and progression to mitosis remains to be determined.

To address whether tethering the pericentromeric chromatin to the nuclear periphery alters gene expression, we analyzed individual embryos expressing ZF-Eme using a microfluidics Biomark approach, which is a robust and quantitative approach amenable to gene expression analysis from low cell number (Supplemental Fig. 4; Guo et al. 2010). We focused specifically on genes that (1) play a role in early development, (2) are activated zygotically between the two-cell and four-cell stages, or (3) are in close proximity to the centromere. The genes analyzed include housekeeping genes, cell cycle-related genes, transcription factors, chromatin modifiers, signaling proteins, developmentally important genes, and genes located at the proximity of the centromere on four different chromosomes $(9,18,19$, and X) (Supplemental Table 1). For the latter group of genes, we performed an in silico search or analyzed genes previously known to be centromere-proximal by cytogenetics: Suv39h1, Suv420h1, Yap1, Gata6, and Rock1. We analyzed levels of expression of all 41 genes simultaneously in 10 biological replicates and three technical replicates. We found no significant changes in gene expression among the noninjected embryos, embryos expressing ZF-GFP, and embryos expressing ZF-Eme (Supplemental Figs. 4, 5).

Finally, we asked whether tethering the MajSats to the nuclear periphery leads to a defective accumulation of heterochromatic marks, which could potentially explain their increased transcriptional activity. For this, we analyzed H3K27me3 and Ring1b in embryos expressing ZF-Eme (Puschendorf et al. 2008; Santenard et al. 2010). Immunostaining revealed that most ZF-Eme embryos display abnormal localization of Ring1b compared with controls (Fig. 4A; Supplemental Fig. 6). We determined the colocalization of MajSats with H3K27me3 and Ring1b in 3D using DNA-FISH and immunostaining followed by 3D reconstruction (Fig. 4A,B). Quantification of the colocalized volume of $\mathrm{H} 3 \mathrm{~K} 27 \mathrm{me} 3 /$ Ring $1 \mathrm{~b}$ within the MajSats revealed a decreased accumulation of the two heterochromatic marks on MajSats in ZF-Eme embryos compared with the controls (Fig. 4C). These data suggest that tethering the MajSats to the nuclear periphery after fertilization impairs the efficient recruitment of these silencing marks to pericentromeric chromatin.

The defect in heterochromatin formation that we report suggests that embryonic nuclear organization is a key factor of epigenetic reprogramming and that the distinctive organization of embryonic nuclei has a regulatory role. The observations that efficiency of cloning upon nuclear transfer is associated with the acquisition of an NLB-like organization (Martin et al. 2006b) further highlight the uniqueness of such reorganization and point toward a necessary step of reprogramming heterochromatin to restore totipotency. 


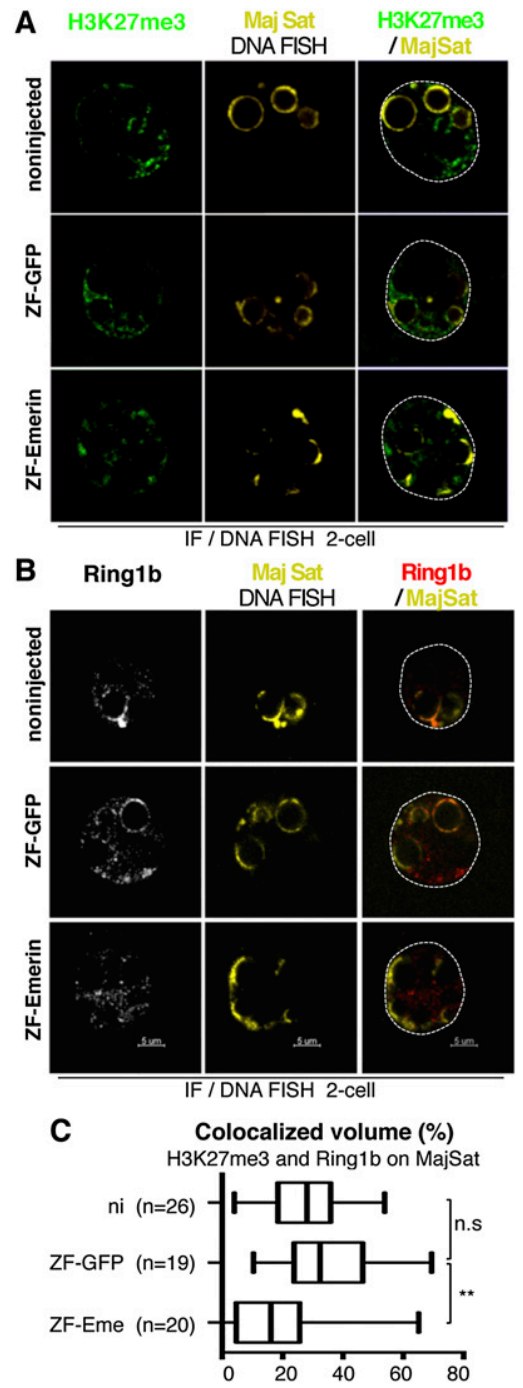

Figure 4. Tethering of pericentromeric chromatin to the nuclear periphery results in decreased accumulation of $\mathrm{H} 3 \mathrm{~K} 27 \mathrm{me} 3$ and Ringlb. $(A, B)$ Representative confocal images of immuno-DNAFISH of early two-cell noninjected, ZF-GFP, and ZF-Eme embryos stained for H3K27me3 $(A)$ and Ringlb $(B)$. DNA-FISH signal for MajSats is in yellow, and dotted lines delineate the nuclear membrane. Shown is a single confocal section from Z-series acquisitions of representative nuclei $(n=26$ embryos for noninjected; $n=19$ embryos for ZF-GFP; $n=20$ embryos for ZF-Eme). Note that for these experiments, DNA-FISH was performed with oligonucleotide probes. (C) Quantification of the volume of MajSats co-occupied by H3K27me3 and Ring1b (in percentage). Whisker plots show the median and the minimum-maximum ranges. (n.s.) Not significant; $\left(^{\star \star}\right) P<0.05$ (ANOVA, Turkey's multiple comparison).

In contrast to the nuclear organization pattern typically found in somatic cells, whereby the most gene-rich chromosomes locate in the center and gene-poor regions are located close to the nuclear periphery (Boyle et al. 2001), studies in bovine embryos revealed that there is no correlation between gene density of chromosome territories and radial positioning prior to the major wave of embryonic genome activation (Koehler et al. 2009). In human cells, relocalization of transgenes to the nuclear periphery alters gene expression (Finlan et al. 2008), but in differentiated cells, the nuclear periphery is believed to be a repressive environment, perhaps through the maintenance of a hypoacetylated chromatin. Our results indicate that the nuclear organization in the early embryo seems to be functionally different from that in differentiated cells.

It remains to be established whether the global dynamics of other chromatin regions within the nuclear space and those of the embryonic chromatin in general further differ from that of somatic cells and whether specific histone modifications would play a role in such regionalization. Our data suggest that the temporal order of events that follow fertilization and the localization of heterochromatin in the 3D nuclear space are tightly regulated and function in parallel to ensure heterochromatic silencing and subsequent development. This adds nuclear reorganization to the molecular cascade of events that dictate establishment of heterochromatin in mammals.

\section{Materials and methods}

\section{Embryo collection}

For microinjection, zygotes were obtained from superovulated F1 females and microinjected at $17 \mathrm{~h}$ post-hCG with in vitro transcribed HA-Eme, ZF-GFP, or Eme-ZF mRNA. Embryos were cultured in KSOM and monitored regularly until fixing for immunostaining, RNA, or DNA-FISH.

\section{FISH}

DNA and RNA-FISH were performed with a MajSat probe covering a full repeat (234p) as described (Miyanari and Torres-Padilla 2012), with minor modifications. Embryos were analyzed in drops to preserve $3 \mathrm{D}$ information.

\section{Immunostaining}

Embryos were fixed $\sim 36-37 \mathrm{~h}$ post-hCG as described (Torres-Padilla et al. 2006). Primary antibodies were anti-H3K27me1 (Millipore), anti-H3K27me3 (Millipore), anti-HP1ß (IGBMC), anti-Lamin B1 (Abcam), anti-HA (Roche), and anti-RING1b (MBL1).

\section{Acknowledgments}

We thank the Institut de Génétique et de Biologie Moléculaire et Cellulaire-Institut Clinique de la Souris imaging facility for support, the sequencing facility for access to the Biomark, B.J. van der Zaal for the PZF:GFO construct, Y. Miyanari for advice on FISH, A. Burton for advice on the Biomark analysis, S. Daujat for advice on ChIP, E. Guccione for support, and A. Bannister for critical reading of the manuscript. M.E.T.-P. acknowledges funding from ANR-09-Blanc-0114, EpiGeneSys Network of Excellence, and European Research Council-Stg "NuclearPotency." J.J. was a recipient of an European Science Foundation Exchange Grant. J.M. acknowledges funding from the Agency for Science, Technology, and Research in E. Guccione's laboratory.

\section{References}

Aguirre-Lavin T, Adenot P, Bonnet-Garnier A, Lehmann G, Fleurot R, Boulesteix C, Debey P, Beaujean N. 2012. 3D-FISH analysis of embryonic nuclei in mouse highlights several abrupt changes of nuclear organization during preimplantation development. BMC Dev Biol 12: 30 .

Akhtar A, Gasser SM. 2007. The nuclear envelope and transcriptional control. Nat Rev Genet 8: 507-517.

Arney KL, Bao S, Bannister AJ, Kouzarides T, Surani MA. 2002. Histone methylation defines epigenetic asymmetry in the mouse zygote. Int $J$ Dev Biol 46: 317-320. 


\section{Jachowicz et al.}

Bengtsson L, Wilson KL. 2004. Multiple and surprising new functions for emerin, a nuclear membrane protein. Curr Opin Cell Biol 16: 73-79.

Boyle S, Gilchrist S, Bridger JM, Mahy NL, Ellis JA, Bickmore WA. 2001. The spatial organization of human chromosomes within the nuclei of normal and emerin-mutant cells. Hum Mol Genet 10: 211-219.

Fadloun A, Eid A, Torres-Padilla ME. 2013. Mechanisms and dynamics of heterochromatin formation during mammalian development: Closed paths and open questions. Curr Top Dev Biol 104: 1-45.

Finlan LE, Sproul D, Thomson I, Boyle S, Kerr E, Perry P, Ylstra B, Chubb JR, Bickmore WA. 2008. Recruitment to the nuclear periphery can alter expression of genes in human cells. PLoS Genet 4: e1000039.

Grewal SI, Elgin SC. 2007. Transcription and RNA interference in the formation of heterochromatin. Nature 447: 399-406.

Guo G, Huss M, Tong GQ, Wang C, Li Sun L, Clarke ND, Robson P. 2010. Resolution of cell fate decisions revealed by single-cell gene expression analysis from zygote to blastocyst. Dev Cell 18: 675-685.

Koehler D, Zakhartchenko V, Froenicke L, Stone G, Stanyon R, Wolf E, Cremer T, Brero A. 2009. Changes of higher order chromatin arrangements during major genome activation in bovine preimplantation embryos. Exp Cell Res 315: 2053-2063.

Kourmouli N, Jeppesen P, Mahadevhaiah S, Burgoyne P, Wu R, Gilbert DM, Bongiorni S, Prantera G, Fanti L, Pimpinelli S, et al. 2004 Heterochromatin and tri-methylated lysine 20 of histone $\mathrm{H} 4$ in animals. J Cell Sci 117: 2491-2501.

Kumaran RI, Thakar R, Spector DL. 2008. Chromatin dynamics and gene positioning. Cell 132: 929-934.

Lindhout BI, Fransz P, Tessadori F, Meckel T, Hooykaas PJ, van der Zaal BJ. 2007. Live cell imaging of repetitive DNA sequences via GFPtagged polydactyl zinc finger proteins. Nucleic Acids Res 35: e107.

Martin C, Beaujean N, Brochard V, Audouard C, Zink D, Debey P. $2006 a$ Genome restructuring in mouse embryos during reprogramming and early development. Dev Biol 292: 317-332.

Martin C, Brochard V, Migne C, Zink D, Debey P, Beaujean N. 2006b. Architectural reorganization of the nuclei upon transfer into oocytes accompanies genome reprogramming. Mol Reprod Dev 73: 11021111.

Miyanari Y, Torres-Padilla ME. 2012. Control of ground-state pluripotency by allelic regulation of Nanog. Nature 483: 470-473.

Nestorov P, Tardat M, Peters AH. 2013. H3K9/HP1 and Polycomb: Two key epigenetic silencing pathways for gene regulation and embryo development. Curr Top Dev Biol 104: 243-291.

Probst AV, Almouzni G. 2011. Heterochromatin establishment in the context of genome-wide epigenetic reprogramming. Trends Genet 27: 177-185.

Probst AV, Santos F, Reik W, Almouzni G, Dean W. 2007. Structural differences in centromeric heterochromatin are spatially reconciled on fertilisation in the mouse zygote. Chromosoma 116: 403-415.

Probst AV, Okamoto I, Casanova M, El Marjou F, Le Baccon P, Almouzn G. 2010. A strand-specific burst in transcription of pericentric satellites is required for chromocenter formation and early mouse development. Dev Cell 19: 625-638.

Puschendorf M, Terranova R, Boutsma E, Mao X, Isono K, Brykczynska U, Kolb C, Otte AP, Koseki H, Orkin SH, et al. 2008. PRCl and Suv39h specify parental asymmetry at constitutive heterochromatin in early mouse embryos. Nat Genet 40: 411-420.

Santenard A, Ziegler-Birling C, Koch M, Tora L, Bannister AJ, TorresPadilla ME. 2010. Heterochromatin formation in the mouse embryo requires critical residues of the histone variant H3.3. Nat Cell Biol 12: 853-862.

Santos F, Peters AH, Otte AP, Reik W, Dean W. 2005. Dynamic chromatin modifications characterise the first cell cycle in mouse embryos. Dev Biol 280: 225-236.

Torres-Padilla ME, Bannister AJ, Hurd PJ, Kouzarides T, Zernicka-Goetz M. 2006. Dynamic distribution of the histone variant H3.3 in the mouse oocyte and preimplantation embryos. Int J Dev Biol 50: 455461. 


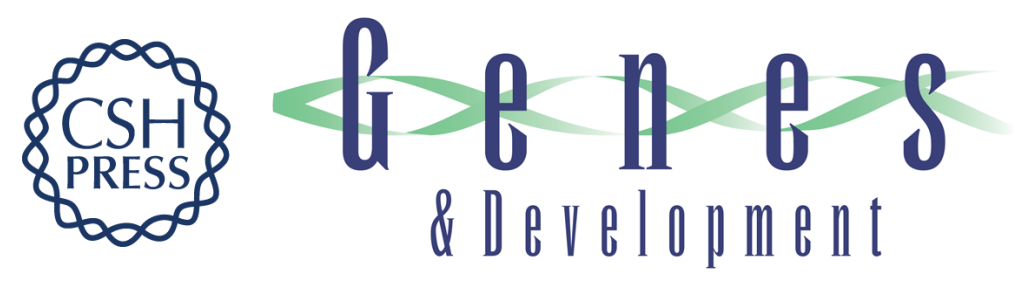

\section{Heterochromatin establishment at pericentromeres depends on nuclear position}

Joanna W. Jachowicz, Angèle Santenard, Ambre Bender, et al.

Genes Dev. 2013, 27:

Access the most recent version at doi:10.1101/gad.224550.113

\section{Supplemental http://genesdev.cshlp.org/content/suppl/2013/11/14/27.22.2427.DC1 \\ Material}

References This article cites 24 articles, 1 of which can be accessed free at:

http://genesdev.cshlp.org/content/27/22/2427.full.html\#ref-list-1

Creative This article is distributed exclusively by Cold Spring Harbor Laboratory Press for the first

Commons six months after the full-issue publication date (see

License http://genesdev.cshlp.org/site/misc/terms.xhtml). After six months, it is available under a Creative Commons License (Attribution-NonCommercial 3.0 Unported), as described at http://creativecommons.org/licenses/by-nc/3.0/.

Email Alerting Receive free email alerts when new articles cite this article - sign up in the box at the top Service right corner of the article or click here.

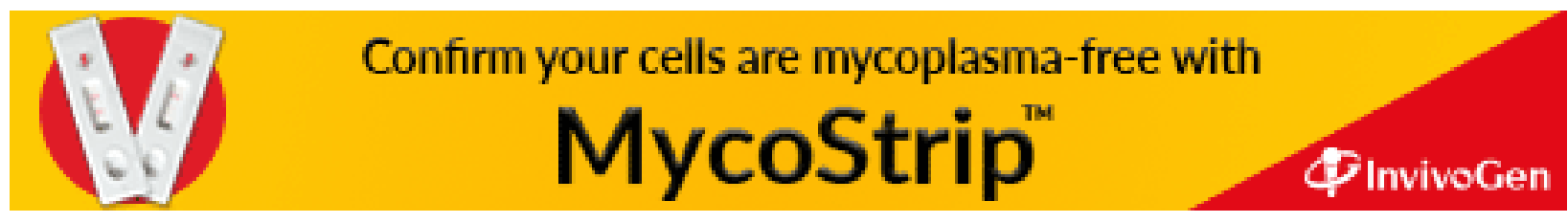

